Osservazioni di Comete

fatte all' Equatoriale di Merz dell' Osservatorio di Torino.

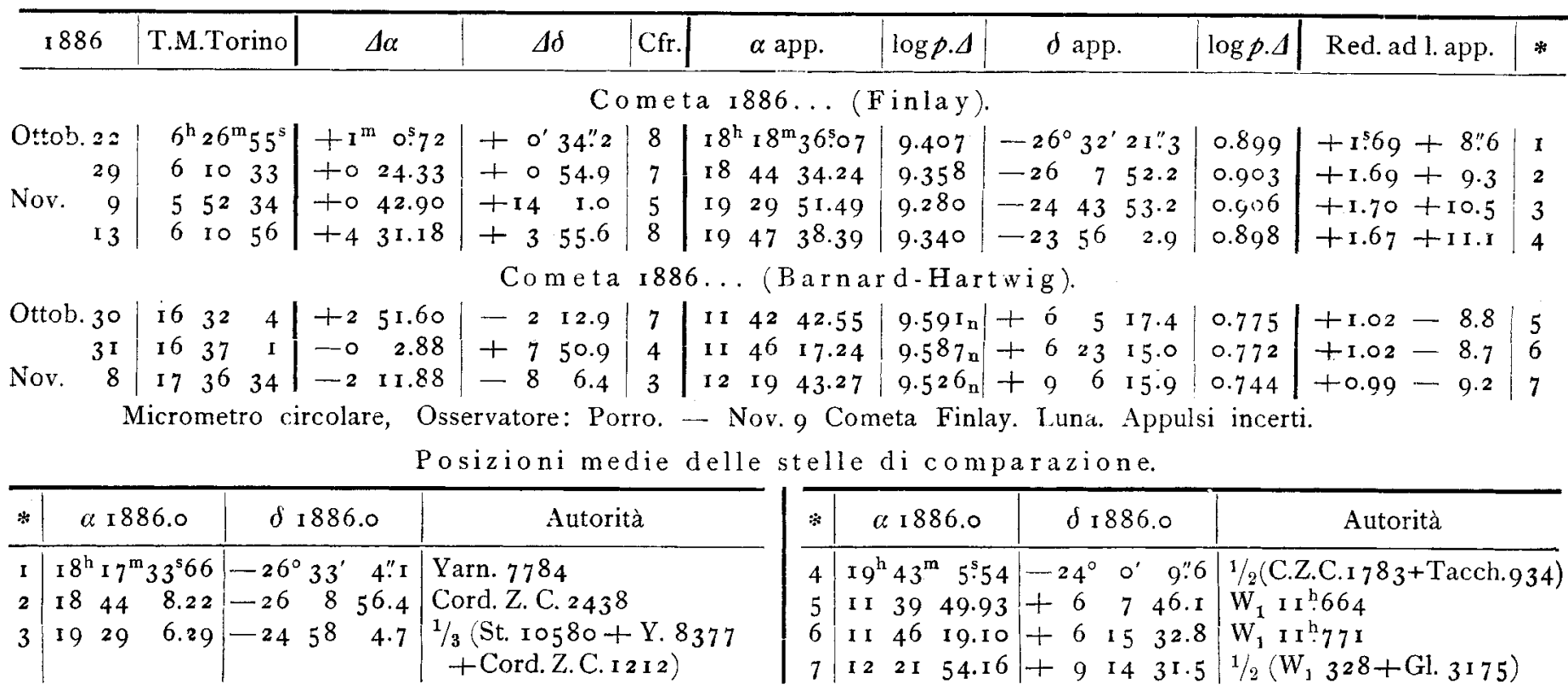

Queste osservazioni furono presentate il 14 Novembre alla R. Accademia delle Scienze di Torino, la quale pubblica i lavori dell' Osservatorio.

F. Porro.

\title{
Ueber die Beobachtung des Planeten (29) Amphitrite in den Münchener Zonen.
}

Für die in A. N. 2749 von Herrn Prof. Schönfeld nachgewiesene Beobachtung der Amphitrite in der Münchener Zone 400 theile ich im Folgenden eine von den früheren Bearbeitungen der Zone völlig unabhängige, und wie ich glaube möglichst scharfe Reduction mit, die auf den Beobachtungen der Zone selbst beruht und nach den Originalnotirungen ausgeführt ist. Der Beobachter war wahrscheinlich Lamont selbst, zum mindesten geht aus einer Notiz hervor, dass er an der Beobachtung des Abends Theil genommen.
Ich wählte 5 hellere, an mehreren Fäden beobachtete Sterne der Zone in der Nähe der fraglichen Beobachtung aus und leitete für dieselben aus anderen Catalogen möglichst zuverlässige Positionen, auf das Auwers'sche System reducirt, ab. Diese Positionen sind mit der Anzahl der benutzten Beobachtungen und der Reduction auf den scheinbaren Ort, in folgender Tabelle zusammengestellt :

\begin{tabular}{|c|c|c|c|c|c|c|c|}
\hline$*$ & $\alpha 1847.0$ & Anz. & Red. & $\delta_{1847.0}$ & Anz. & Red. & Autorität \\
\hline $\mathbf{I}$ & $20^{\mathrm{h}} 37^{\mathrm{m}} \times 7^{\mathrm{s}} \cdot 39$ & 36 & +3.71 & $-22^{\circ} 3^{\prime} 5^{6 . \prime 4}$ & 33 & +20.5 & Br., Pi., Tayl., Y., 9 yr. \\
\hline 2 & 2059 & 3 & +3.70 & $-22 \quad 5^{6} \quad 44.6$ & 3 & +21.6 & $\mathrm{AOe}_{\hat{2}}$, Yarn \\
\hline 3 & $2143^{6.01}$ & 5 & +3.69 & -232312.0 & 5 & $+21 \cdot 9$ & Ll., $\mathrm{AOe}_{2}$, Yarn. \\
\hline 4 & $21 \quad 14 \quad 13.72$ & 29 & +3.67 & $-2319 \quad 6.4$ & 26 & $+22 \cdot 3$ & Pi., Tayl., $\mathrm{AO}_{2}, \mathrm{Y}$., Stone \\
\hline 5 & 212250.01 & 4 & +3.64 & $-2240 \quad 48.0$ & 5 & +22.7 & $\mathrm{AOe}_{2}$, Yarn. \\
\hline
\end{tabular}

und ergeben, wenn man dieselben in der gewöhnlichen Weise als Fundamentalsterne für die Reduction der Zone benutzt, als Reductionselemente die folgenden Zahlen:

$$
\begin{aligned}
& \text { * Uhrcorrect. Gew. Decl. Nullpunkt Gew. }
\end{aligned}
$$

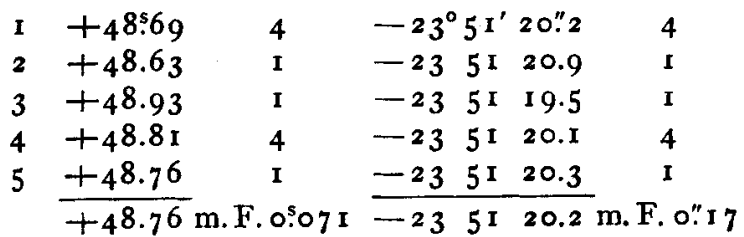

Die Gewichte sind nach Maassgabe der Sicherheit der obigen Positionen, sowie der Anzahl der beobachteten
Fäden vertheilt. Als mittlerer Fehler eines einzelnen FadenAntrittes ergiebt sich aus denselben Sternen 0.047. Collimationsfehler und Decl. der Axe sind, als von verschwindendem Einfluss, nach den früheren Bestimmungen beibehalten worden, dagegen der Run des Zonenbogens und die Refractionen neu gerechnet.

Als scheinbarer Ort der Amphitrite folgt hieraus:

$$
\alpha \text { app. } \quad \delta \text { app. Par. }
$$

I 847 Aug. I $21^{\mathrm{h}} G^{\mathrm{m}} 59^{\mathrm{s}} .2 \mathrm{I}-23^{\circ} 29^{\prime} 42^{\prime \prime} 8 \quad+5^{\prime \prime} 34$,

nur unerheblich von der früheren, von Herrn Prof. Schönfeld angeführten Position abweichend.

München 1886 Oct. ıо. Dr. F. Bauschinger. 